\title{
Arquitectura estructural y estratigráfica de secuencia pre-aptiense de la cuenca oriente ecuatoriana
}

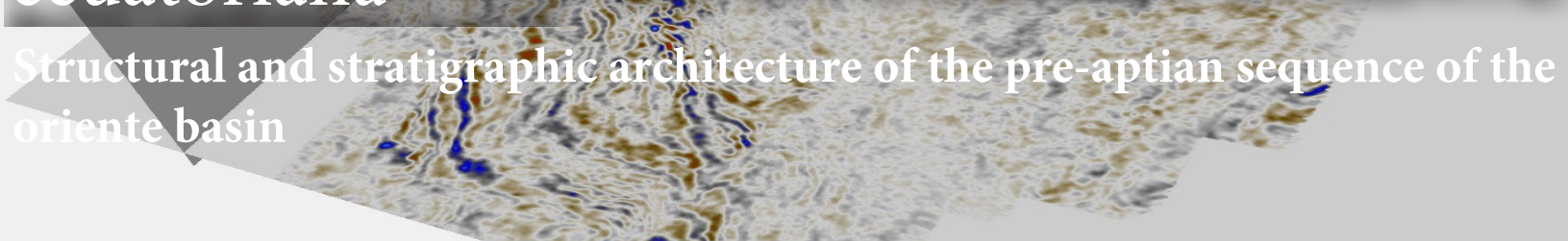

Angulo Andrea

e-mail: andriu_2909@hotmail.com

Bustos Jairo

e-mail: jgbustos@uce.edu.ec
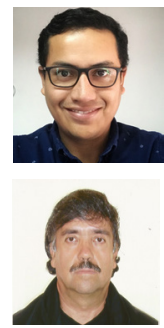

\section{Rivadeneira Marco}

Ministerio de Hidrocarburos

e-mail: mrivadeneira80@gmail.com

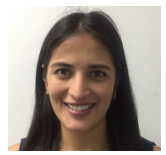

Navarrete Johanna

Docente Universidad Central

e-mail: janavarrete@uce.edu.ec

\section{Baby Patrice}

Géoscience Environnement Toulouse

e-mail: patrice.baby@ird.fr

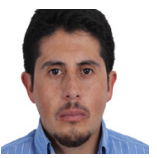

\section{Resumen}

La configuración estructural y estratigráfica de la secuencia pre-Aptiense en la Cuenca del Oriente, se deriva del desarrollo de dos eventos tectónicos superpuestos. El primero pertenece a la aparición de un régimen extensional durante el Jurásico Medio-Valanginiense, representado por depresiones de hemi-graben y graben que constituyen los tres dominios tectónicos de la cuenca. En el graben Sacha-Shushufindi, el relleno sedimentario son representados por sedimentos volcaniclásticos continentales de la Formación Chapiza, parcialmente contemporáneos con la actividad del arco volcánico Jurásico Misahuallí (Tschopp, 1953), que se desarrolla en el margen occidental de la cuenca. Probablemente, la extensión está vinculada a los períodos de retroceso de un slab que se evidencia por el desarrollo de una zona de subducción y un control extensional en la región de arco posterior. El segundo episodio representa la inversión de fallas normales a inversas producidas a partir de una tectónica de compresión, lo que resulta en mega estructuras positivas (Anticlines Sacha y Shushufindi). La edad de la inversión podría estar relacionada con la época volcánica Hauteriviense de la Formación Chapiza Superior o Miembro Yaupi, que se desarrollan en una sola operación contra la estructura invertida de Sacha.

\section{Palabras clave: pre-aptiense; formación chapiza; extensión trasarco; inversión tectónica}

\section{Abstract}

The structural and stratigraphic configuration of the pre-Aptian sequence in the Oriente Basin, is derived from the development of two overlapping tectonic events. The first, belongs to the apperarance of an extensional regime during the Middle Jurassic-Valanginian, represented by half-graben and graben depressions that constitute the three tectonic domains of the basin. At the Sacha-Shushufindi graben the sedimentary filling are continental volcaniclastic sediments of the Chapiza Formation, partially contemporaneous whit the activity of the Jurassic Misahualli volcanic arc (Tschopp, 1953), that is developed in the western margin of the basin. Probably the extension is linked to salb roll-back periods by the development of a subduction zone and an extensional control in the back-arc region. The second episode represents the inversion of normal to inverse faults produced from a compressive tectonics, which results in mega positive structures (Sacha and Shushufindi An ticlines). The age of the inversion could be related to the Hauterivian volcanic of the Upper Chapiza Formation or Yaupi Member, which are developed in onlap against the Sacha inverted structure. 


\section{Introducción}

La secuencia estratigráfica pre-Aptiense de la Cuenca Oriente se encuentra constituida por una cubierta sedimentaría marino-continental del Paleozoico-Mesozoico, superpuesta a un basamento cratónico pre-Cámbrico (Díaz et al., 2014). Está caracterizada por una arquitectura estructural relacionada a la inversión de un sistema de fallas extensionales, que han fracturado la cuenca en tres dominios tectónicos: Faja Plegada y Corrida Subandina (FPCS), Corredor Sacha-Shushufindi (CSS), y Sistema Invertido Capirón Tiputini (SICT) (Baby et al., 2013).

En el presente estudio, se examinó la configuración estratigráfica y estructural principalmente de la zona norte del CSS, misma que permite comprender el desarrollo de los eventos tectónicos, extensivo y compresivo, que actúan durante el pre-Aptiense. De esta forma, adicionalmente se contribuye con un modelo tectono-estratigráfico más acertado y aporta a la comprensión de un posible sistema petrolífero en el pre - cretácico ecuatoriano.

\section{Material y métodos}

Para el desarrollo de la investigación, se consideró nueva información de sísmica 3D y de pozos: una franja orientada N-S de varios cubos sísmicos (1.930 Km2), posicionados en los campos petroleros más importantes de la cuenca (Libertador, Shushufindi y Sacha); y el levantamiento estratigráfico de dos pozos: IP-17 y BE x-1 localizados en la FPCS en el campo Pungarayacu (Figura.1), complementado con análisis bioestratigráficos y radiocronológicos llevados a cabo en los laboratorios de Petroamazonas EP, y Australian National University para dos muestras datadas por

U-Pb SHRIMP en circones, una en cada pozo.

\section{Resultados}

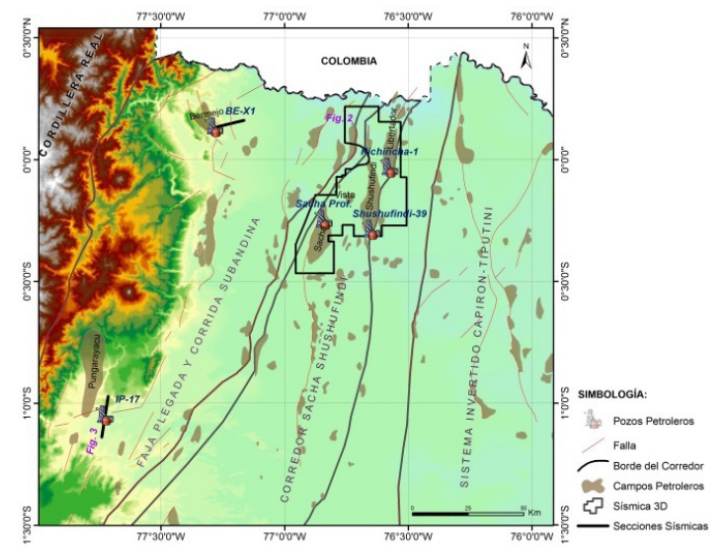

Figura 1. Esquema de perforación.

Se definieron seis unidades sismo-estratigráficas, "USE", agrupadas desde el punto de vista de evolución tectónica - estratigráfica en tres estadios de desarrollo estratigráfico: 1) pre-extensional, 2) sin-extensional y 3) sin-inversión.

\section{Estratigrafía pre-extensional}

Consiste de las unidades geológicas que preceden el desarrollo de un sistema extensivo.

- USE-1-Basamento: está caracterizado por una configuración caótica y difusa, sin reflexiones dominantes (Fig. 2a, 2b y 3). Su litología consiste de rocas ígneas y metamórficas del Escudo Cratónico pre-Cámbrico Guayano-Brasileño (Tschopp, 1953; Díaz et al., 2014).

- USE-2-pre-Pumbuiza: muestra reflectores dispuestos paralelamente, con alta amplitud y continuidad, marcando el inicio de la subsidencia y primera sedimentación depositada sobre el basamento. Esta unidad no ha sido atravesada por ningún sondeo, pero probablemente se correlacione con la Fm. Contaya en Perú, constituida de lutitas graptolíticas intercaladas con areniscas del Ordovícico (Spencer, 1973). Al norte del CSS, se evidencia el desarrollo de posibles facies arcillosas o salífera, que deforma la secuencia suprayacente, posterior a su sepultamiento. En total se identifican 3 grandes cuerpos, estos representan probablemente a diapiros, dispersos bajo el campo Vista (Fig. 2a). Esta unidad está ausente en la FPCS, así lo demuestra el registro litológico del pozo IP-17 (Fig. 4) y la sísmica 2D (Fig. 3). Probablemente se limita al borde oeste del CSS y se extiende hacia el oriente.
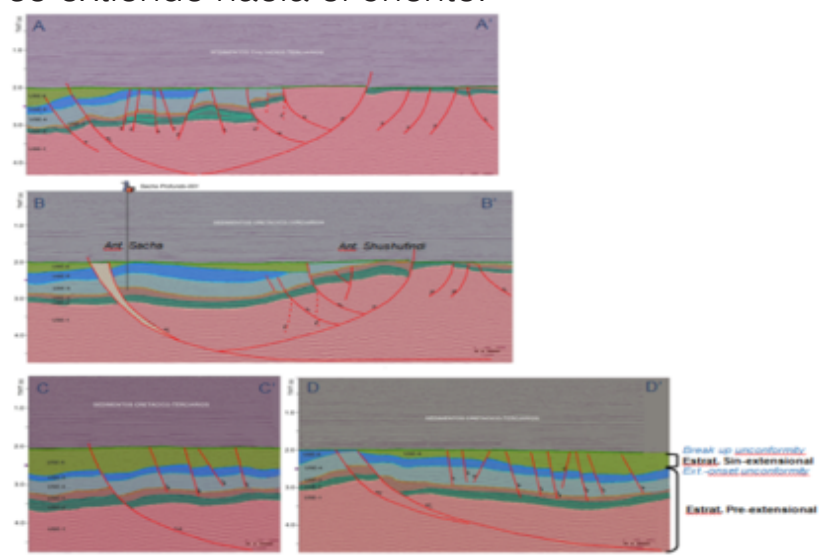

Figura 2a Tres secciones sísmicas transversales y una longitudinal al CSS ilustran la geometría de la secuencia pre-Aptiense y de sus cuerpos sedimentarios.

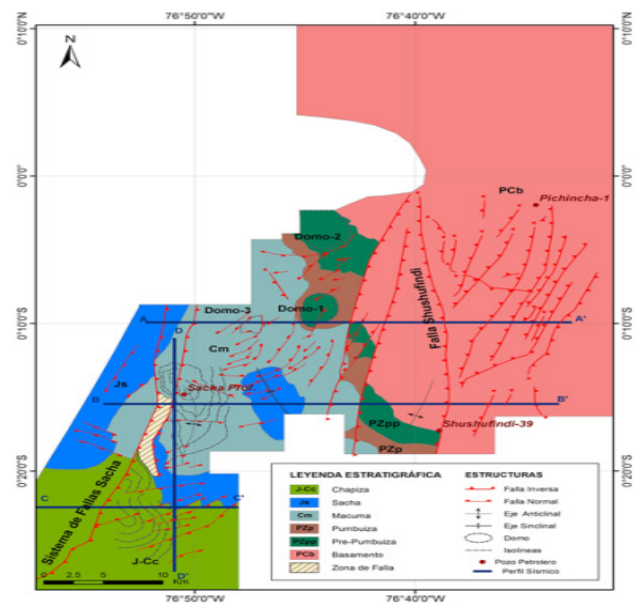

Figura 2b: mapa geológico de subsuelo (-2505 ms) de la sección norte del CSS. 
- USE-3-Pumbuiza: sus reflectores registran menores amplitudes y media a baja continuidad (Fig. 2 y 3). Su litología ha sido descrita por los pozos Sacha Profundo y Shushufindi-039 en el CSS, que está constituida de areniscas y lutitas del Devónico. En la FPCS, los ripios del pozo IP-17 y $B E X$ - 1 definen un paquete homogéneo de lutitas, con rangos de TOC entre 0.5-2 \% (Regular), Kerógeno tipo III con calidad pobre a regular proveniente de restos vegetales terrestres depositados en ambientes marino-someros (Fig. 4)

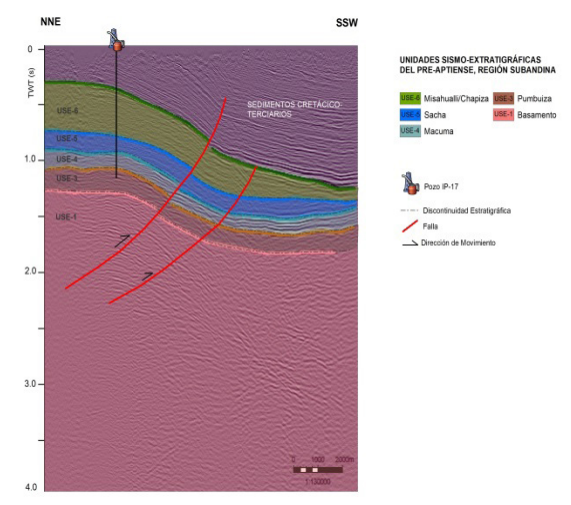

Fig. 3: Sección sísmica longitudinal a la FPCS (parte sur, ver Fig.1), mostrando un anticlinal asociado al desarrollo de fallas inversas, atravesada por el pozo. IP-17.

USE-4-Macuma: registra un cuerpo de reflectores claros y continuos dispuestos paralelamente considerado como una plataforma carbónica, con potentes estratos de caliza bioclástica y dolomitas, intercaladas de lutitas, limolitas y areniscas finas del Pensilvánico-Pérmico (Díaz et al., 2014). Hacia la región Subandina, los estratos mayormente calcáreos, pasan a ser intercalados en la base con facies volcano-sedimentarias de origen aún desconocido (Fig. 4).

- USE-5-Sacha: muestra un pequeño onlap contra la superficie subyacente, por encima continúa con un arreglo paralelo a divergente de baja continuidad y moderada amplitud (Fig. 2b). Dentro del pozo Sacha Prof., se constituye de una secuencia de capas rojas, datadas en su límite inferior del Triásico Tardío (Rivadeneira y Sánchez,

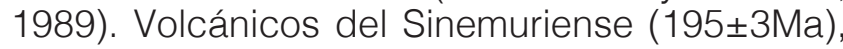
datados en el presente estudio por U-Pb SHRIMP en circones, fueron encontrados en los pozos IP17 y BE X-1, correspondiendo cronológicamente al Ciclo Santiago/Sacha (Fig. 4). La base de este ciclo en ambos dominios tectónicos no presenta la misma edad, por lo que se puede interpretar un límite diacrónico más joven hacia la FPCS.

\section{Estratigrafía sin-extensional}

Las características de relleno de la USE-6, considerada como la Fm. Chapiza, definen a esta unidad como una secuencia sin-extensional, que se acomoda sobre una marcada superficie estratigráfica, extensional-onset unconformity, misma que define el límite con la secuencia pre-extensional (Fig. 2-C y D). Esta unidad se compone de una alternancia de reflectores de alta a baja amplitud y continuidad, configurados estructuralmente en una cuña de material sin-extensional, asociado al basculamiento y movimiento del bloque colgante sobre el fallamiento normal principal a vergencia este que controla la subsidencia.

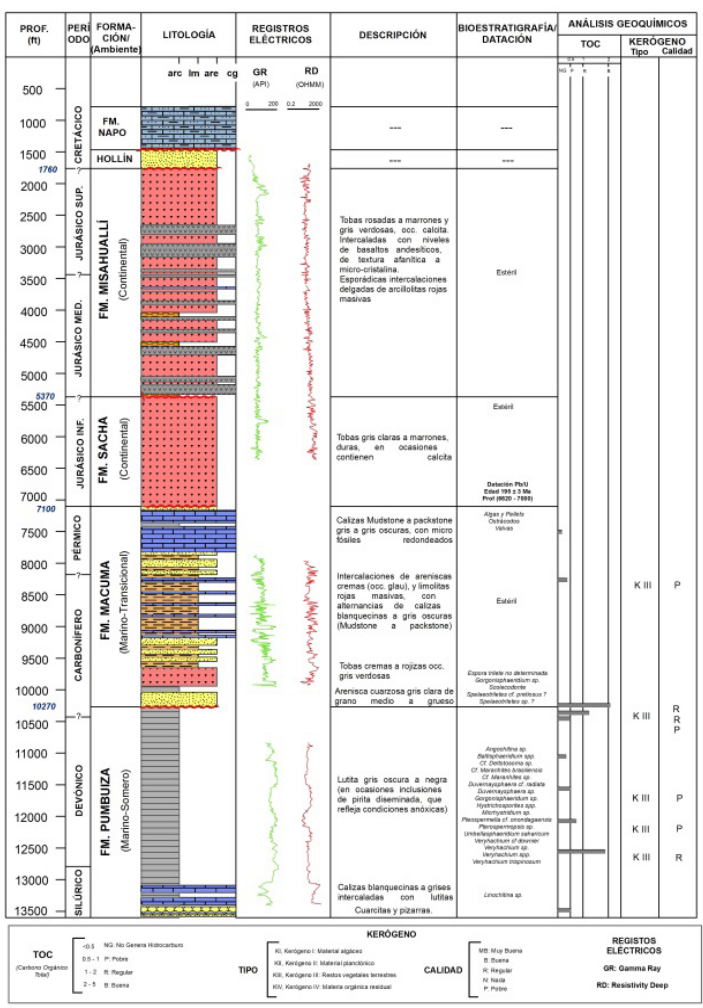

Fig. 4: Columna litológica, nuevas dataciones y niveles de roca madre de la secuencia pre-Aptiense del pozo IP-17.

Esta secuencia comprende una espesa serie de capas rojas y sedimentos volcanoclásticos continentales, considerados parcialmente coetáneos con la actividad del arco magmático jurásico Misahuallí (Tschopp, 1953). Con estas consideraciones y en base al contacto estratigráfico con la Fm. Sacha del Triásico Superior-Jurásico Inferior (Rivadeneira y Sánchez, 1989), se designa una edad del Jurásico Medio, para el inicio de la sedimentación Chapiza y por tanto el inicio de la extensión.

\section{Estratigrafía sin-inversión}

El registro litológico del pozo Sacha Prof. señala tobas y basaltos gris obscuros al tope de la Fm. Chapiza o Miembro Yaupi, datados por palinología y radiometría en los ríos Yaupi y Chapiza de $132 \mathrm{Ma}$ (Hauteriviense), lo que los diferencian de los volcánicos Misahuallí (Jaillard, 1997). Sus características geoquímicas y significado geodinámico son aun inciertos, pero en base al desarrollo de onlaps contra la estructura invertida Sacha (Fig. 2b), se presume que marquen la edad de una inversión, probablemente desarrollada entre el 
Hauteriviense y Barremiense, anterior a la depositación de la Fm. Hollín.

\section{Estructura y evolución tectónica}

La región norte del CSS (Fig. 2) expone el desarrollo de un sistema extensivo, con una configuración estructural tipo graben, cuyo relleno sedimentario, lo constituyen los depósitos de la Fm. Chapiza. Está controlado en sus márgenes, por el Sistema de fallas Sacha al oeste y falla Shushufindi al este, de tipo lístricas y dirección de inclinación opuesta, que delinean su geometría y orientación NE-SO. Fallas normales planas están acentuadas entre los márgenes del graben, con diferencia de estilos estructurales entre un segmento norte y un segmento sur. Al norte, el rumbo de las fallas es subparalelo con respecto al eje principal del graben y su buzamiento es hacia el NO; mientras que al sur, presentan una orientación oblicua al eje principal, con buzamientos hacia el SE (Fig. 2).

Las fallas principales de carácter lístrico que controlaron los márgenes del graben, fueron reactivadas durante una tectónica compresiva, provocando el desplazamiento de las secuencias pre y sin-extensionales, que dan resultado a las mega estructuras positivas, correspondientes a los anticlinales Sacha y Shushufindi (Fig. 2b).

\section{Estructuras extensivas preservadas o con débil inversión}

Los controles estructurales vigentes durante la etapa extensional, están mayormente preservados al sur del campo Sacha, donde la estructura original de la extensión se ha conservado sin mayores alteraciones. La sección sísmica exhibe una estructura extensional sin-sedimentaria, anticlinal de rollover, que muestra el desarrollo del flexuramiento del bloque sedimentario por encima de la falla lístrica Sacha. Los sedimentos sin-extensionales (USE-6) descienden y rellenan el espacio producido durante la apertura de la falla, formando un cuerpo convexo. Fallas normales planas sintéticas se acentúan a intervalos de tiempo regulares durante la extensión, y deforman el rollover.

\section{Estructuras invertidas}

Las estructuras Sacha y Shushufindi, que representan los máximos del desplazamiento inverso, consisten en anticlinales formados por la propagación de las fallas del borde durante la inversión en su movimiento. La parte superior de las estructuras muestra una contracción neta, mientras que en profundidad mantienen signos de extensión (Figura 2-B). Están alineadas paralelamente al eje de la cuenca, alcanzando longitudes de alrededor de $20 \mathrm{Km}$, y $6 \mathrm{Km}$ de ancho (Fig. 2). Si bien ambos márgenes de la cuenca presentan el mismo estilo de deformación, el margen oriental registra un mayor levantamiento, generando durante la cinemática de contracción la aparición de fallas inversas antitéticas al fallamiento Shushufindi, que levanta la secuencia y decapita antiguas fallas normales (Fig. 2b). Por detrás, se genera un retrocorrimiento controlado por fallas transpresivas, las mismas que probablemente formen parte de una gran estructura en flor (Baby et al., 2013).

En la FPCS, la sísmica 2D que atraviesa el pozo IP-17 (Fig. 3) manifiesta el desarrollo de un anticlinal, en respuesta a la inversión del movimiento de la falla normal previa, que controló la depositación de la secuencia sin-extensional Chapiza. El movimiento de falla contraccional desplazó la secuencia sin y pre-extensional sobre el bloque superior fallado, por encima de la elevación regional predeformada (Fig. 3).

\section{Modelo de evolución}

La interpretación estratigráfica y estructural de la sísmica 3D expuesta anteriormente, permitió reconstruir el modelo de evolución para la secuencia pre-Aptiense dentro del CSS, durante la etapa de extensión y posterior compresión, desarrolladas en el Jurásico Medio-Cretácico Inferior. Explicado en la Figura 5.

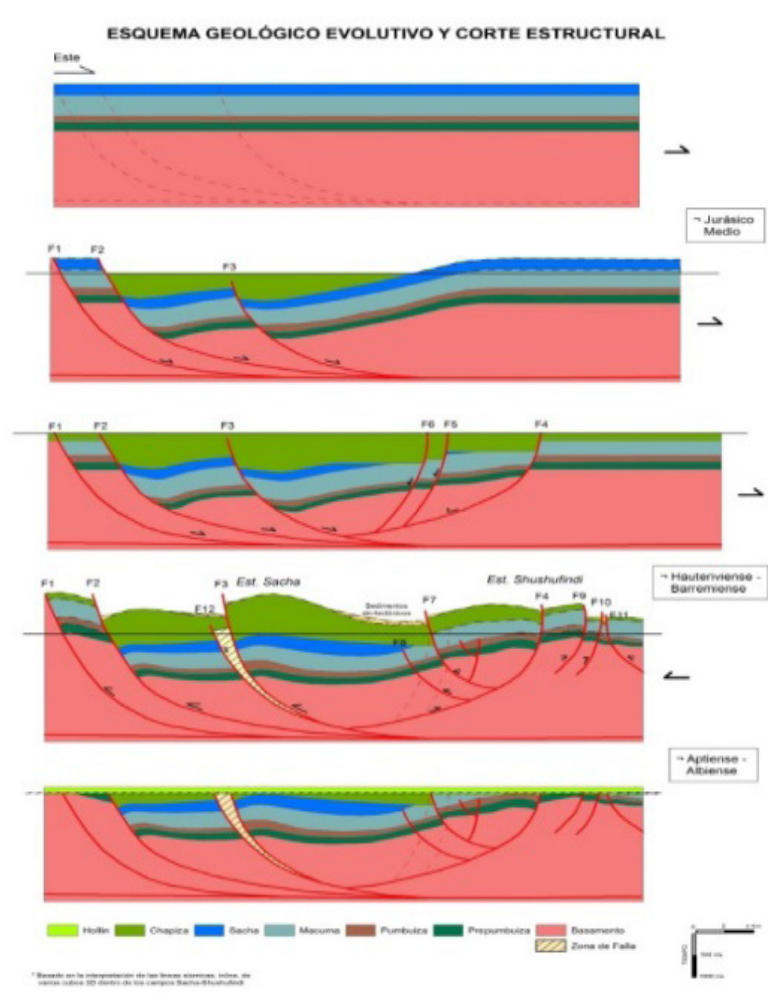

Fig. 5: Esquema geológico evolutivo de la cinemática de deformación entre el Jurásico Medio y el Cretácico Inferior, en el CSS.

El inicio de la activa extensión ocurrió aproximadamente en el Jurásico Medio, con el desarrollo de un fallamiento extensional lístrico en el borde occidental durante la depositación de la Fm. Chapiza. En esta 
etapa, actuaron fallas lístricas sintéticas (F1, F2 y F3), involucrando el basamento, que se unen en profundidad en una superficie de despegue, con sentido de desplazamiento hacia el oriente. El movimiento de falla, produce un fuerte basculamiento de la secuencia sedimentaria por encima del fallamiento lístrico, lo que da origen a una geometría de semi-graben, con desarrollo de estructuras extensionales sin-sedimentarias de tipo anticlinal de rollover, preservadas al sur del campo Sacha (Fig.2-B).

Con el aumento de la extensión, la geometría inicial evolucionó a un graben, desarrollando fallas normales antitéticas al fallamiento lístrico principal; encabezada por la falla Shushufindi (F4), que representa el borde oriental del graben, seguida de fallas escalonadas con similar dirección de movimiento (F5 y F6), que avanzan hacia el interior del graben. La sedimentación muestra un incremento en su extensión, rellenando todo el graben.

En el Hauteriviense aproximadamente, las fallas directas que controlaron el desarrollo inicial de la cuenca invierten su movimiento y levantaron toda la secuencia sedimentaria, manteniendo signos de extensión en profundidad y mostrando contracción asociada a crecimiento de anticlinales hacia la superficie (estructura Sacha y Shushufindi). Con la deformación progresiva, durante el levantamiento del borde este de la cuenca sobre la falla Shushufindi, se desarrollan fallas antitéticas (F7 y F8), que cortan las fallas normales F5 y F6 impidiendo su reactivación; además del desarrollo de un retrocorrimiento que se propaga hacia el este (F10, F11 y F12).

Para el Aptiense, después de un periodo de erosión, la cuenca es sellada por depósitos clásticos fluviales, correspondientes a la Fm. Hollín (Baby et al., 2013).

\section{Discusión}

A partir de la interpretación estructural y estratigráfica de los datos sísmicos y de los pozos seleccionados, se estableció un control tectónico extensional durante la depositación de la Fm. Chapiza, a la que se asigna una edad Jurásico Medio y Cretácico Inferior, sobre la base de su posición estratigráfica. Con ello se reevalúa y posteriormente se invalidó la edad Triásico Tardío-Jurásico Temprano de una extensión, antiguamente propuesta sobre la base de la definición de una supuesta depositación sin-rift de las Fms. Sacha/Santiago, descrito en el CSS y al sur de la Región Subandina propuesta por Díaz, 2014.

La extensión probablemente de edad Jurásico Medio y Valanginiense, es desarrollada en forma concomitante a una zona de subducción, definida sobre la base del arco volcánico Misahuallí del Liásico Tardío y Jurá- sico Superior, ubicado al oeste de la Cuenca Oriente (Romeuf et al., 1997). Este importante elemento geotectónico lleva a la idea de un sistema extensional de tipo back-arc, cuyo desarrollo probablemente esté ligado a periodos de slab roll-back, en el cual el slab de subducción retrocede y se verticaliza (Martinod, et al. 2006), provocando la propagación de una extensión detrás del arco, que deja como resultado los sistemas de semi-grabenes y grabenes que forman los tres dominios tectónicos.

En la FPCS, la fuerte tectónica transpresiva a la que ha sido sometida, por su posición en la zona de wedge-top de la actual cuenca de foredeep Oriente (Baby et al., 2013), ha modificado en su mayoría las evidencias de extensión, resultando complejo su caracterización. En el CSS, las estructuras preservadas la definen como un graben, relleno de depósitos volcano-sedimentarios de la Fm. Chapiza, considerados como facies proximales al arco Misahuallí (Díaz et al., 2014). Hacia el este, las evidencias extensionales se conservan en importantes semi-grabenes, conocidos dentro del SICT. El más representativo es el semi-graben Tambococha, cuyo relleno sin-extensional, consiste en depósitos marino-someros del Jurásico Tardío-Neocomiense definidos como la Fm. Tambococha, la misma que es considerada equivalente lateral a la $\mathrm{Fm}$. Chapiza y atribuida a facies distales del arco (Díaz et al., 2014).

El cierre del sistema extensional, marcado por la discordancia angular regional a la base de la Fm. Hollín, probablemente se desarrolla en el Hauteriviense-Barremiense, inferido por los basaltos al tope de la Fm Chapiza de $132 \mathrm{Ma}(\mathrm{K} / \mathrm{Ar})$, asociados a un cambio geodinámico (Jaillard, 1997), y desarrollados en onlap contra la estructura positiva Sacha. Probablemente la compresión que generó la inversión del sistema extensivo, este relacionada a la propagación hacia el oeste de la ruptura Tethys de Laurasia y Gondwanna durante la apertura inicial del Océano Atlántico, expresando deformación tectónica en el borde del Pacífico (Jaillard et al., 1995). Las cuencas de Acre de Brasil y Solimões invertidas en el Jurásico Tardío durante la Orogenia Juruá (Caputo, 2014), coinciden con la gran compresión y cizallamiento en los países andinos, por lo que se podría atribuir a la orogenia brasileña Juruá (Caputo, 2014).i)

\section{Conclusiones}

La arquitectura estructural y disposición estratigráfica de la secuencia pre-Aptiense a lo largo de la sección norte del CSS, responde al desarrollo de una cuenca extensional, activa durante la depositación de la Fm. Chapiza (Jurásico Medio-Valanginiense). Este perio- 
do estuvo controlado por un sistema de fallas lístricas (S. de fallas Sacha al oeste y Shushufindi al este), que delinearon la orientación y geometría de la cuenca, formando una depresión alargada tipo graben con rumbo preferentemente NE-SW.

Anticlinales asociados a movimientos contraccionales (Anticlinales Sacha y Shushufindi), en los bordes de la cuenca, manifiestan una inversión tectónica posterior (Hauteriviense-Barremiense), probablemente vinculada a los volcánicos de la parte superior de la Fm. Chapiza de 132 Ma (Hauteriviense) (Hall \& Calle in Jaillard, 1997), considerada por Jaillard (1997) como el Miembro Yaupi, que se desarrollan en onlap contra la estructura Sacha.

En forma concomitante con el desarrollo del régimen distensivo, en la Región Subandina se desarrollaba el arco volcánico Misahuallí (Liásico Tardío y Jurásico Superior) (Romeuf, et al., 1997; Jaillard, 1997), que permite inferir un sistema extensional de backarc que evolucionó detrás del arco magmático. Probablemente los tres dominios tectónicos estuvieron controlados por un régimen extensional de amplia distribución detrás del arco volcánico Misahuallí, ligado a periodos de slab rollback.

La inversión tectónica podría estar relacionada con la propagación hacia el oeste de la ruptura Tethys, durante la apertura inicial del Océano Atlántico expresando deformación tectónica en el continente, conocida como la Orogenia Juruá, en Brasil (Caputo, 2014).

El levantamiento estratigráfico en los pozos IP-17 y BE X-1 muestran una continuación lateral de las unidades del pre-Aptiense, a excepción de la Fm. Pre-

Pumbuiza. El registro sedimentario empieza con la Fm. Pumbuiza en inconformidad sobre el basamento. Los estratos mayoritariamente calcáreos de la Fm. Macuma pasan a ser intercalados por volcanosedimentos hacia la FPCS. Volcánicos del Sinemuriense (Jurásico Inferior), datados por U-Pb SHRIMP en circones, sobreyacen a la Fm. Macuma, en ambos pozos, cronológicamente se correlacionan con el Ciclo Santiago/Sacha (Triásico Superior-Jurásico Inferior). La base de este ciclo no presenta la misma edad en ambos dominios tectónicos, por lo que se puede considerar un límite diacrónico más joven hacia la FPCS. El Ciclo Misahuallí/ Chapiza, sin-extensional, es mucho más potente en la FPCS por su cercanía con el arco volcánico Misahuallí.

\section{Referencias biliográficas}

Baby, P., Rivadeneira, M., Barrragán, R., \& Christophoul, F. (2013). Thick-skinned tectonics in the Oriente foreland basin of Ecuador. Geological So- ciety, London, Special Publications, 377, 59-76.

Caputo, M. V. (2014). Juruá Orogeny: Brazil and Andean Countries. Brazilian Journal of Geology, 44(2), 181-190.

Díaz, M., Baby, P., Rivadeneira, M., \& Christophoul, F. (2014). El pre-Aptiense en la Cuenca Oriente Ecuatoriana. En P. Baby, M. Rivadeneira, \& R. Barragán, La Cuenca Oriente: Geología y Petróleo (págs. 31-50). Quito: Travaux de l'Institut Francais d'Études Andines.

Jaillard, E. (1997). Síntesis Estratigráfica y Sedimentológica del Cretáceo y Paleógeno de la Cuenca Oriental del Ecuador. Informe Final, Convenio Orstom-Petroproducción, Quito.

Jaillard, E., Sempére, T., Soler, P., Carlier, G., \& Marocco, R. (1995). The role of Thethys in the evolution of the Northen Andes between Late Permian and Late Eocene times. En A. Nairn, L. Ricou, B. Vrielynck, \& J. Dercourt, The Ocean Basins and Margins: 8. The Tethys Ocean (págs. 463-492). New York: Plenum Press.

Martinod, J., Espurt, N., Guillaume, B., Funiciello, F., Faccenna, C., \& Farías, M. (2006). Control de la velocidad de placas y de la densidad del slab sobre la dinámica de la subducción oceánica y el régimen tectónico de la placa superior: Análisis de modelos análogos. XI Congreso Geológico Chileno, 2, 275-278.

Rivadeneira, M., \& Sánchez, C. (1989). Consideraciones geológicas del Preaptense de la Cuenca Oriente. Sexto Congreso Ecuatoriano de Ingeniería en Geología, Minas y Geotecnia, 214-252.

Romeuf, N., Munch, P., Soler, P., Jaillard, E., Pik, L., \& Aguirre, R. (1997). Mise en évidence de 2 lignées magmatiques dans le volcanisme du Jurassique inférieur de la zone subandine équatorienne. Comptes Rendus Academie of Sciences, 324, 361-368.

Spencer, A. (1973). Geological Survey of the Rio Santa Catalina to Contaya Dome area. Lima, Perú.

Tschopp, H. (1953). Oil explorations in the Oriente of Ecuador. Quito: AAPG Bulletin. 